\title{
"Second Mind" una aplicación móvil para el almacenamiento de memorias del usuario
}

Fecha de recepción: 2021-10-28 • Fecha de aceptación: 2021-12-14 • Fecha de publicación: 2022-02-10

Roberth Alexander Almache Vallejos ${ }^{1}$ Comando Conjunto de las Fuerzas Armadas del Ecuador

roberth9501@hotmail.com

https://orcid.org/0000-0002-5947-9576

Jorge Ramiro Castro Chavez ${ }^{2}$

Int Food Services Corp, Ecuador

jorgesuzuky666@gmail.com

https://orcid.org/0000-0003-4500-5940

Diego Alejandro González Calderon ${ }^{3}$

Rankmi, Chile

dgonzalez.jim@gmail.com

https://orcid.org/0000-0003-0121-867X

Johanna Daniela Ruano Cardenas ${ }^{4}$

Maint.SA, Ecuador

danyruanoc13@gmail.com

https://orcid.org/0000-0002-7766-2809

\section{RESUMEN}

Actualmente, a nivel profesional y personal se maneja una gran cantidad de información de cualquier ámbito, sea laboral, escolar, familiar, salud, etc., y por el avance tecnológico ya no se llevan registros manuales, sino un almacenamiento digital, ubicándolo en varios lugares, tales como correos, notas rápidas o archivos de texto, que limitan la obtención inmediata de información a recordar. En este sentido, la presente investigación tiene como objetivo diseñar un prototipo móvil 
llamado "Second Mind", que permite al usuario almacenar información (notas, tips, conceptos, rutinas, ayudas técnicas, etc) en el dispositivo móvil, donde se puede buscar y tener accesibilidad a datos importantes o relevantes, permitiendo manejar su información de forma efectiva, inmediata y organizarla en base a su necesidad, con esto se le permitirá accionar sin dificultad en cualquier lugar que se desempeñe con sus registros personales.

\title{
PALABRAS CLAVE: digital, app, almacenamiento, tecnológico, dispositivo
}

\begin{abstract}
Currently, at a professional and personal level, a large amount of information is handled in any field, be it work, school, family, health, etc., and due to technological advances, manual records are no longer kept, but digital storage, locating it in various places, such as emails, quick notes or text files, which limit the immediate collection of information to be remembered. In this sense, the present research aims to design a mobile prototype called "Second Mind", which allows the user to store information (notes, tips, concepts, routines, technical aids, etc) in the mobile device, where you can search and have accessibility to important or relevant data, allowing you to manage your information effectively, immediately and organize it based on your need, with this will allow you to act without difficulty anywhere you perform with your personal records.
\end{abstract}

KEYWORDS: digital, app, storage, technological, device 


\section{Introducción}

En el día a día, para poder comunicarnos y realizar incluso distintas acciones, se necesita de información o datos que faciliten las actividades diarias. Se ha visto que en generaciones anteriores la tecnología no era muy accesible y manejaban su información en libros, libretas y registros manuales, poco a poco la tendencia ha ido cambiando y se ha permitido digitalizar la información, facilitando incluso hasta la escritura de la misma. Se observa actualmente que incluso con este avance, y con varios aplicativos que se tiene, como por ejemplo en Google el Dropbox, MEGA, MediaFire, Google Drive, que se lanzó en el año 2012, así como también el mismo bloc de notas, o el correo electrónico (Martín-Barbero, 2015), existen limitantes al obtener los datos deseados en el momento justo que se requiere.

La información muchas veces se almacena en varios lugares y registros, de hecho, sea por capacidad, disponibilidad, o netamente por falta de una aplicación como repositorio que ayude a almacenarla, ha dificultado la disponibilidad, ya que con frecuencia se necesita traer un dato importante a la mente, provocando retrasar o posponer ciertas actividades, sea a nivel personal, comercial, de ingeniería, e incluso a nivel escolar, se suelen suspender actividades académicas por no disponer de información inmediata que permita organizar al usuario y trabajar de forma productiva.

En base a dicha necesidad, se ha visto como mejoramiento la creación de un prototipo de aplicación móvil en Xamarin forms, manejando una base de datos SQLite y con el IDE de desarrollo Visual Studio .Net 2019, desarrollada por versiones en GitHub (B, 2021), la misma que permitirá al usuario almacenar información, sean textos, imágenes, links en su dispositivo con un buscador que con solo ingresar una palabra clave se obtendrá la información, manteniendo disponibilidad continua e inmediata.

Con lo antes expuesto, este desarrollo está dirigido a cualquier persona que maneje y disponga de un dispositivo móvil Android, y tenga la necesidad de registrar datos y tener memorias almacenadas para su desempeño, siendo un aplicativo muy útil y novedoso donde puede llegar a muchos usuarios, con tan solo aprovechar la facilidad móvil (Dominguez, 2018). Este desarrollo es un aporte a la sociedad que puede llegar a cualquier parte y sin distinción, a tal punto de que a un niño se le facilite almacenar información escolar y a un adulto memorias difíciles de recordar y comprender con tan solo una búsqueda en su dispositivo.

\section{Metodología}

La implementación de una metodología en un desarrollo de software proporciona grandes beneficios al proyecto, mejorando la calidad y productividad dentro del entorno de trabajo, para este caso en específico se utilizó la observación directa y la metodología Kanban.

De acuerdo con Marshall y Rossman (1989), citados en Kawulich (2005), la observación es "la descripción sistemática de eventos, comportamientos y artefactos en el escenario social elegido 
para ser estudiado" (p.79), en este sentido, el uso de esta técnica permitió tener un panorama más amplio en cuanto a la descripción de las situaciones que se presentaron.

A su vez, es importante me mencionar la definición de la metodología aplicada. "La palabra Kanban viene del japonés y traducida literalmente quiere decir tarjeta con signos o señal visual. El tablero más básico de Kanban está compuesto por tres columnas: "Por hacer", "En proceso" y "Hecho". Si se aplica bien, y funciona correctamente, sirve como una fuente de información, ya que demuestra dónde están los cuellos de botella en el proceso y qué es lo que impide que el flujo de trabajo sea continuo e ininterrumpido", (Kanbanize, s.f).

\subsection{Fases de ciclo de producción}

Kanban está basado en el desarrollo incremental, esto quiere decir que el trabajo a desarrollar se divide en diversas fases, con esto lo que se busca es acelerar el proceso del desarrollo del software; estas fases son puestas en las columnas que correspondan. En estas tarjetas no se describe la totalidad de la tarea a realizar solamente, se da una descripción general del entorno de lo que se quiere, para que el equipo de trabajo conozca la carga que posee; en estas tarjetas se describen si esta tarea depende de otras (Gilibets, 2020).

En la siguiente Tabla 1 se visualizará la lista de requerimientos presentados para este proyecto. 
Tabla 1.

Requerimientos del prototipo

\begin{tabular}{lll}
\hline Requerimientos funcionales & \\
\hline Identificación & Nombre del requerimiento & Descripción del requerimiento \\
\hline RF01 & $\begin{array}{l}\text { Recursos de prototipo móvil } \\
\text { "Second Mind" }\end{array}$ & $\begin{array}{l}\text { Para el prototipo se debe emular desde un } \\
\text { celular. } \\
\text { El acceso al aplicativo mantendrá un login. }\end{array}$ \\
\hline RF02 & $\begin{array}{l}\text { Menú de opción de prototipo } \\
\text { móvil }\end{array}$ & $\begin{array}{l}\text { La aplicación tendrá dos botones, uno de } \\
\text { registro para ingreso de datos y otro para } \\
\text { consulta de la información. }\end{array}$ \\
\hline RF03 & Registro de información de & $\begin{array}{l}\text { El usuario al seleccionar Registro podrá } \\
\text { ingresar y grabar los datos como: textos, } \\
\text { imágenes, links en la prototipo y una palabra } \\
\text { clave para recordar. }\end{array}$ \\
& & $\begin{array}{l}\text { El usuario al seleccionar en pantalla } \\
\text { "Recordar" se habilitará un buscador, al } \\
\text { tipear una palabra clave podrá mostrar su } \\
\text { información almacenada en "Second Mind". }\end{array}$ \\
\hline RF04 & Consulta de información en & prototipo "Second Mind" \\
& & $\begin{array}{l}\text { El usuario podrá eliminar información } \\
\text { registrada que ya no desee mantener } \\
\text { almacenada. }\end{array}$ \\
\hline RF05 & Eliminar Información en & \\
& prototipo móvil &
\end{tabular}

Requerimientos no funcionales

\begin{tabular}{lll}
\hline Identificación & Nombre del requerimiento & Descripción del requerimiento \\
\hline RNF01 & Interfaz del sistema. & $\begin{array}{l}\text { El prototipo presentará una interfaz de usuario } \\
\text { sencilla para que sea de fácil manejo a los } \\
\text { usuarios. }\end{array}$ \\
\hline RNF02 & Cámara para prototipo móvil & $\begin{array}{l}\text { Se requiere uso de cámara propia del } \\
\text { dispositivo móvil. }\end{array}$ \\
\hline Identificación & Nombre del requerimiento & Descripción del requerimiento \\
\hline RNF03 & Almacenamiento en prototipo & $\begin{array}{l}\text { La memoria permitirá que la búsqueda sea } \\
\text { altamente inmediata, los datos se almacenan } \\
\text { en SQLITE y las imágenes se almacenarán en } \\
\text { Amazon para AW S3 "repoawsxamarin". }\end{array}$ \\
\hline RNF04 & Desempeño de prototipo móvil & $\begin{array}{l}\text { Se garantizará a los usuarios un desempeño } \\
\text { en cuanto a los datos almacenado en el } \\
\text { sistema ofreciéndole una confiabilidad a la } \\
\text { misma. }\end{array}$ \\
\hline &
\end{tabular}

\subsection{Requerimientos de software}

- GitHub. - Es un servicio basado en la nube que aloja un sistema de control de versiones (VCS) llamado Git. Éste permite a los desarrolladores colaborar y realizar cambios en proyectos compartidos, a la vez que mantienen un seguimiento detallado de su progreso (González, 2021). 
- Visual Studio .Net 2019.- Lenguaje de programación diseñado para crear una gran variedad de aplicaciones que se ejecutan en .NET. Este lenguaje con seguridad de tipo es eficaz y están orientado a objetos. Se basan en .NET Compiler Platform "Roslyn", que proporciona API de análisis de código sofisticadas y es de código abierto en GitHub (Microsoft, s.f).

- Xamarin Forms. - Es un marco de interfaz de usuario de código abierto que permite a los desarrolladores crear aplicaciones de Xamarin.Android, Xamarin.iOS y Windows desde una única base de código compartida.

- BD SQLite. - Es un ligero motor de bases de datos de código abierto, que se caracteriza por mantener el almacenamiento de información persistente de forma sencilla (SQLite,2018). A diferencia de otros sistemas gestores de bases de datos como MySQL, SQL Server y Oracle $\mathrm{DB}, \mathrm{SQLite}$ tiene las siguientes ventajas:

- No requiere el soporte de un servidor, SQLite no ejecuta un proceso para administrar la información, si no que implementa un conjunto de librerías encargadas de la gestión.

- No necesita configuración, libera al programador de todo tipo de configuraciones de puertos, tamaños, ubicaciones, etc.

- Usa un archivo para el esquema, crea un archivo para el esquema completo de una base de datos, lo que permite ahorrarse preocupaciones de seguridad.

- Es de código abierto: está disponible al dominio público de los desarrolladores, al igual que sus archivos de compilación e instrucciones de escalabilidad (Develou, s.f).

Es por eso que SQLite es una tecnología cómoda para los dispositivos móviles. Su simplicidad, rapidez y usabilidad permiten un desarrollo muy amigable.

- Xamarin Essentials. - Es un paquete compatible con Microsoft que se conecta a varias características nativas para el usuario y mejora con cada versión, en este caso se usó para las imágenes y links (Ruiz, 2017).

- AWS SDK. - Para .NET simplifica el uso de los servicios de AWS porque ofrece un conjunto de bibliotecas que a los desarrolladores que usan. NET les resultan lógicas y familiares (Madhuri \& Madhuri, 2016). Todos los AWS SDK ofrecen compatibilidad con el ciclo de vida de las API, como administración de credenciales, reintentos, cálculo de referencias de datos y serialización. AWS SDK para .NET también admite abstracciones de nivel superior, como S3 Transfer Utility, Cognito Identity Provider y AWS DyanamoDB Session State Provider. Visite el GitHub de aws/dotnet y encuentre bibliotecas y herramientas para .NET en AWS (Amazon, s.f).

\subsection{Restricciones}

Tomando en cuenta que el proyecto tiene como propósito un prototipo funcional, se tiene en cuenta las siguientes restricciones: 
- Para el prototipo no se podrá usar dispositivos móviles que tengan una versión inferior de API 22.

- El prototipo será compatible desde la versión 5.1 o posterior.

- El dispositivo que utilice el prototipo debe tener conexión a Internet para almacenar las imágenes en el repositorio de Amazon AWS.

- En el prototipo no se podrán almacenar videos.

A continuación, en la Tabla 2 se muestran las personas involucradas en el desarrollo del proyecto con sus role asignados:

Tabla 2.

Responsables y roles en la metodología Kaban

\begin{tabular}{ll}
\hline Personas/Instituciones & Roles \\
\hline Mg. Renato Toasa/Universidad Israel & Stakeholder \\
\hline Diego González & Service Request Manager. \\
\hline Jorge Castro/Robert Almache/Johanna Ruano & Service Delivery Manager. \\
\hline
\end{tabular}

A su vez, se estructura un cronograma de actividades como base, para el desarrollo del proyecto que se muestra a continuación en la Figura 1.

Figura 1.

Cronograma de actividades

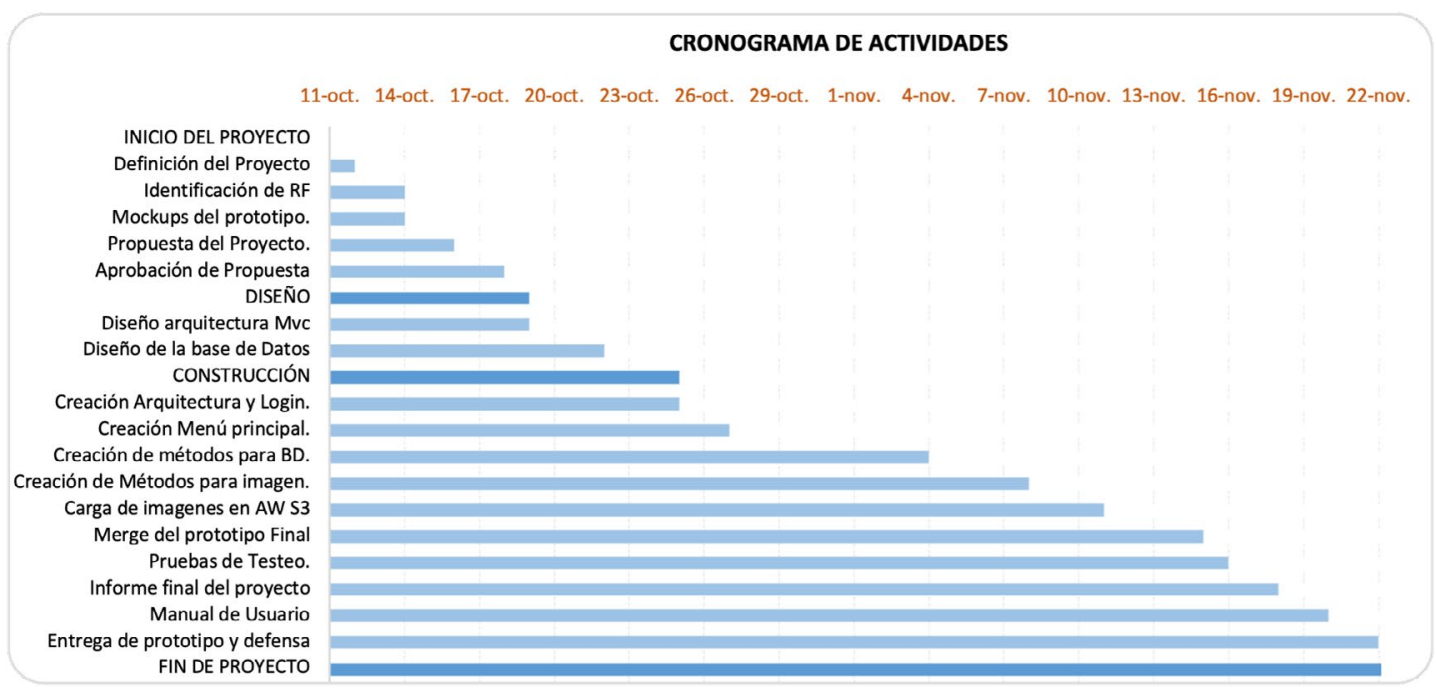




\subsection{Fases del proyecto}

\section{- Fase 1.- Inicio}

En esta fase desarrollada del 11 al 19 de octubre se definieron los lineamientos del proyecto, el tema, la identificación de requerimientos, mockups del prototipo y gestión de actividades para el desarrollo.

En la siguiente Figura 2 se muestra el tablero Kanban, definido por actividades del proyecto, tareas por hacer, tareas en progreso y tareas finalizadas.

Figura 2.

Planificación e inicio actividades Kanban

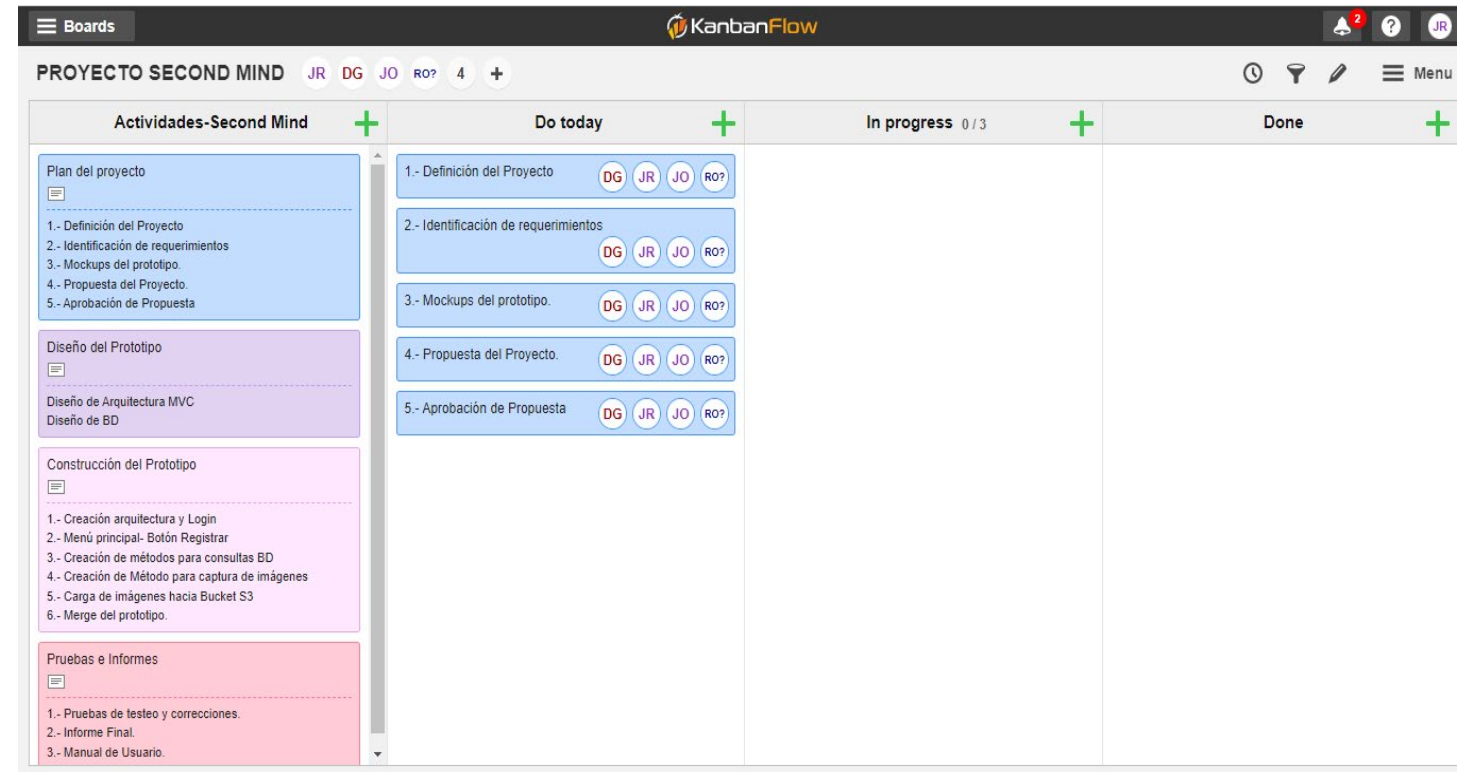

\section{- Fase 2.- Diseño del prototipo}

En esta fase, distribuida del 19 al 23 de octubre, se procedió con el diseño y desarrollo del prototipo, en base a los requerimientos funcionales y no funcionales identificados.

En ella se registra y se ejecutan las siguientes actividades que se visualizan en la Figura 3. 
Figura 3.

Tablero fase 2

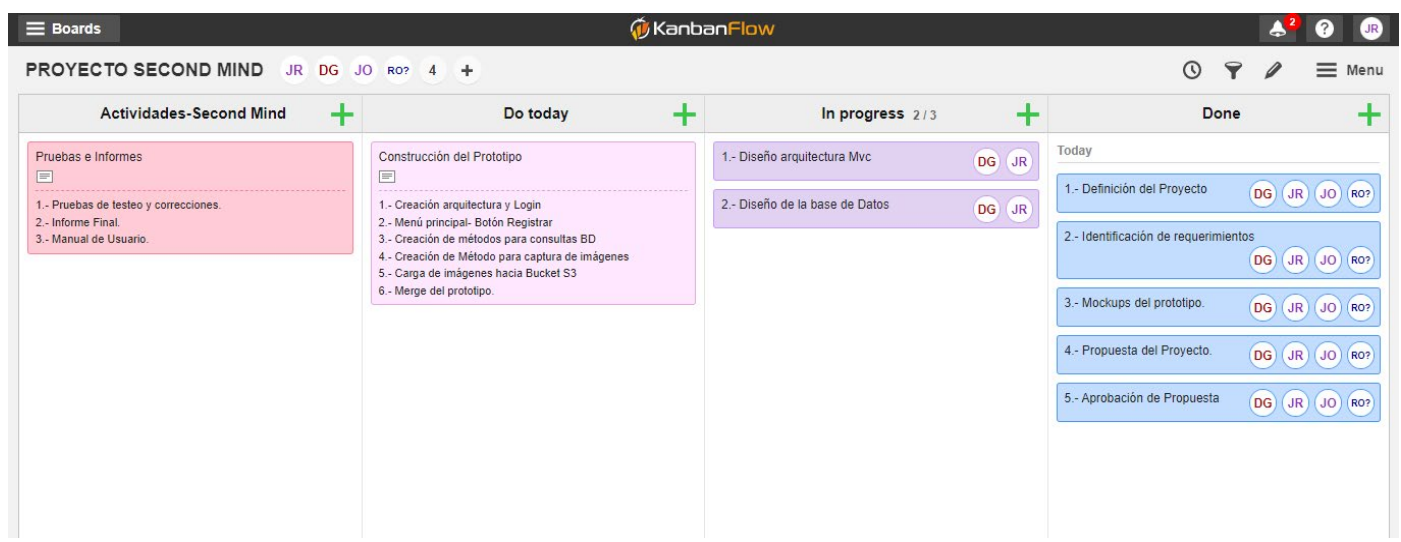

En la siguiente Figura 4 se muestra el diseño de la arquitectura MVC

Figura 4.

Arquitectura Mvc "Second Mind"

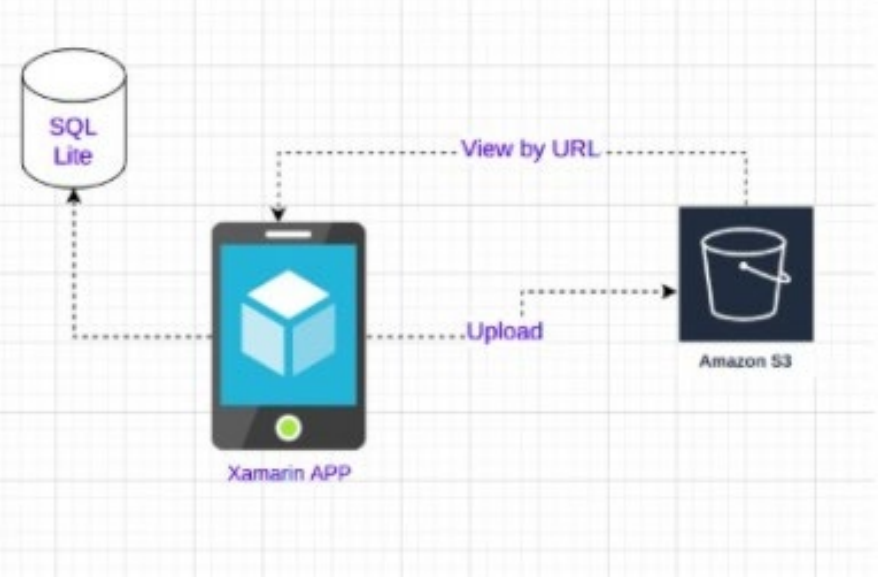

\section{- Fase 3.- Construcción del prototipo}

En esta fase, distribuida del 25 de octubre al 15 de noviembre, se procedió con la creación de Login, menú principal donde consta el botón Registrar/Consultar, métodos para BD e imagen, cargas de imágenes y Merge. En la Figura 5 se observa el tablero correspondiente. 
Figura 5.

Tablero fase 3

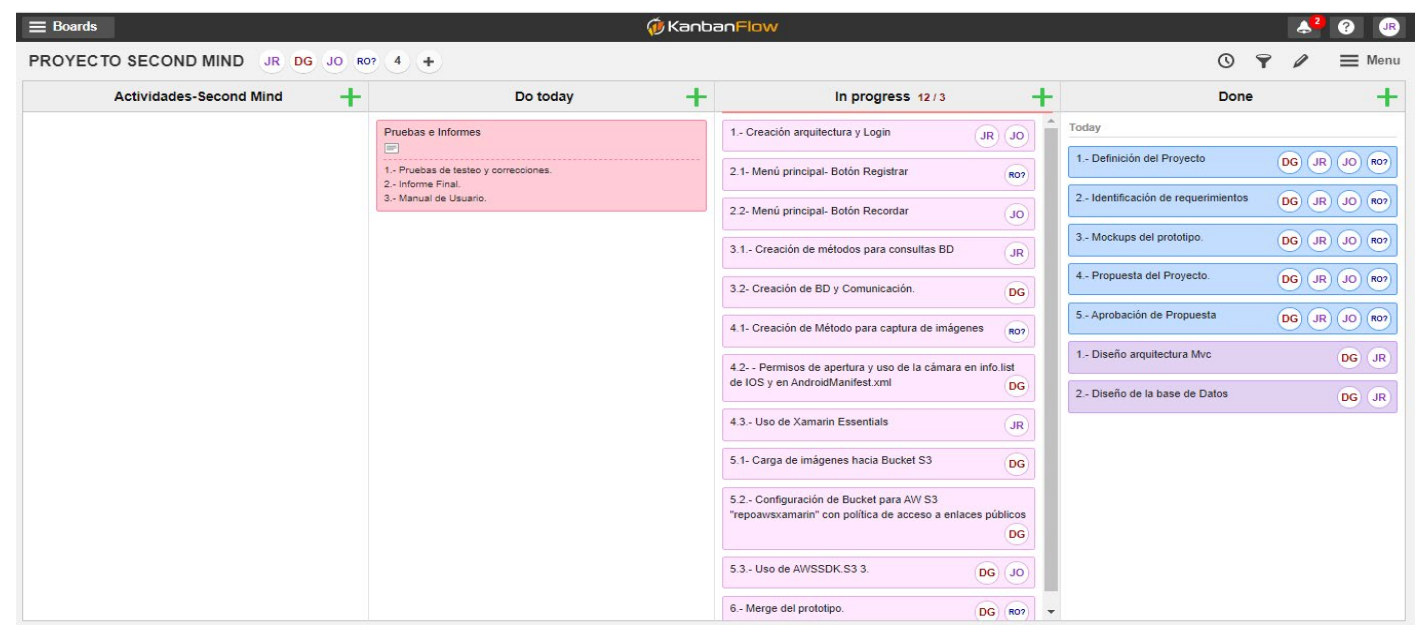

\section{- Fase 4.- Pruebas e informes}

En esta fase, distribuida del 15 de noviembre al 21 de noviembre, se realizaron las pruebas de testeo y correcciones del prototipo, el informe final y el manual de usuario (Figura 6).

Figura 6.

Tablero fase 4

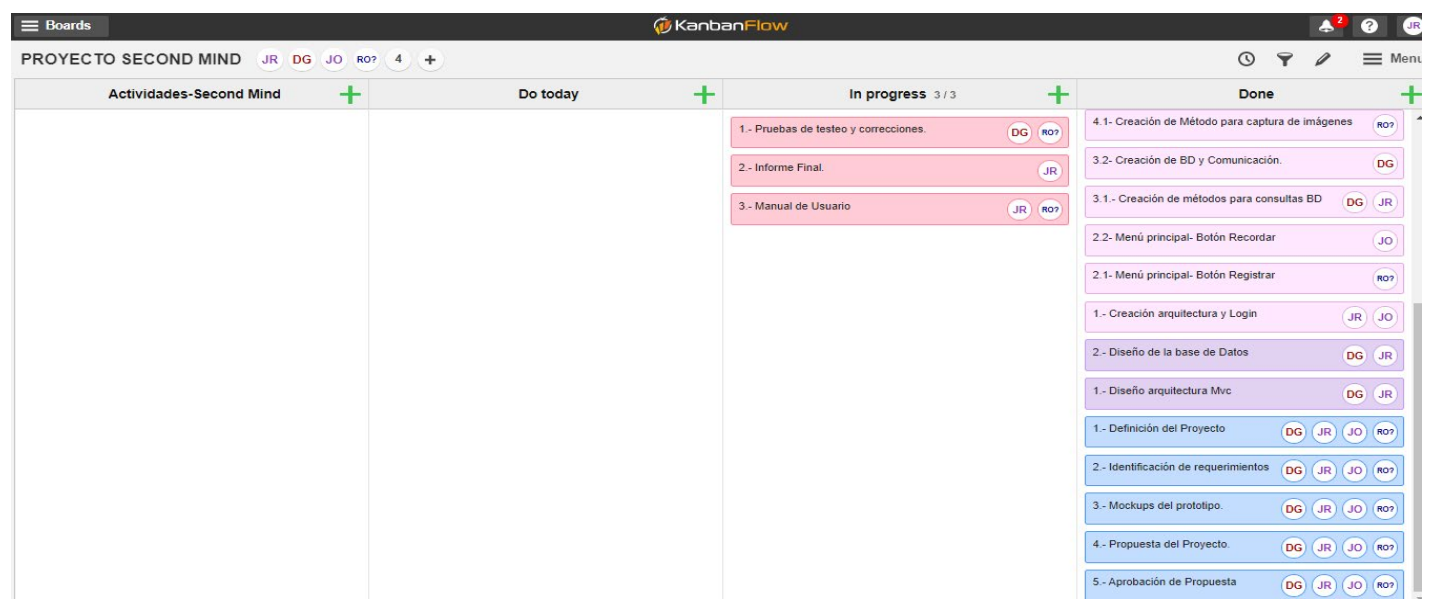

De esta forma, en las fechas estipuladas en el cronograma se da por concluidas las actividades (Figura 7), usando la metodología desarrollada por intervalos y con responsables por cada actividad o tarea. 
Figura 7.

Fin actividades en Tablero Kanban

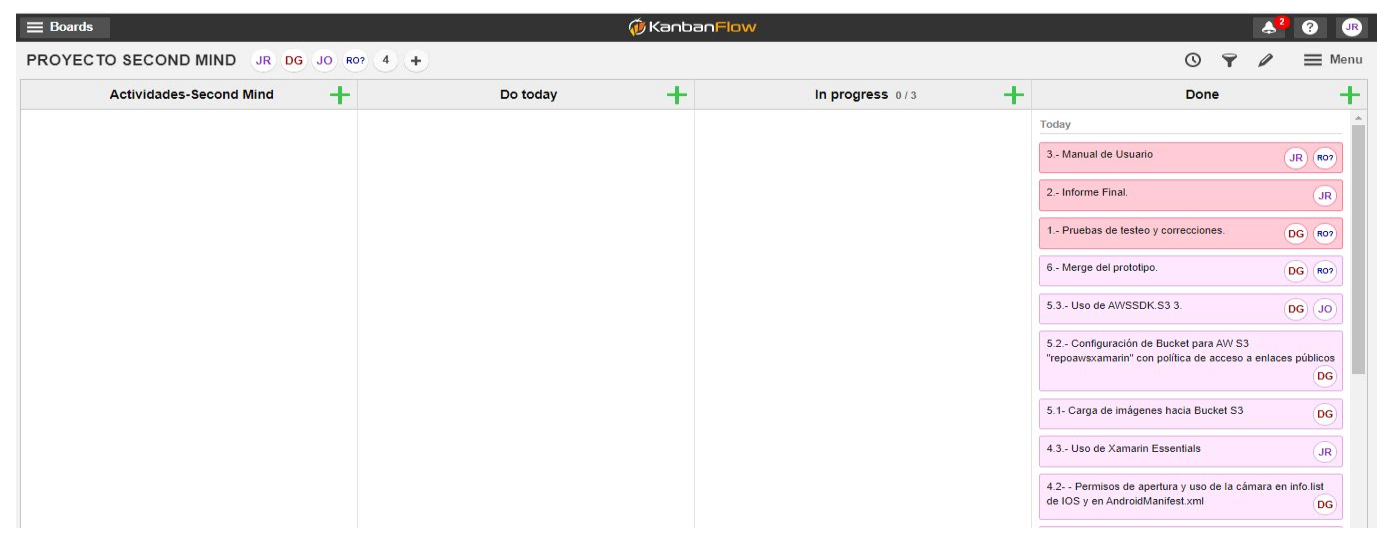

\section{Resultados}

Para el estudio se tiene como parte de los resultados la funcionalidad del prototipo móvil que cumple con las especificaciones planteadas y aprobadas, el mismo se ve reflejado y demostrado con las pruebas funcionales, que a continuación se detallan: 
En las Figura 8 y Figura 9 se observa la pantalla principal y login único (administrador).

Figura 8.

Login de User
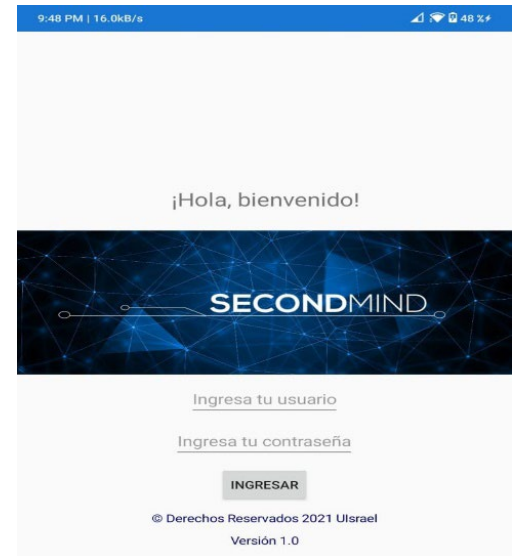

Figura 9.

Pantalla Inicial

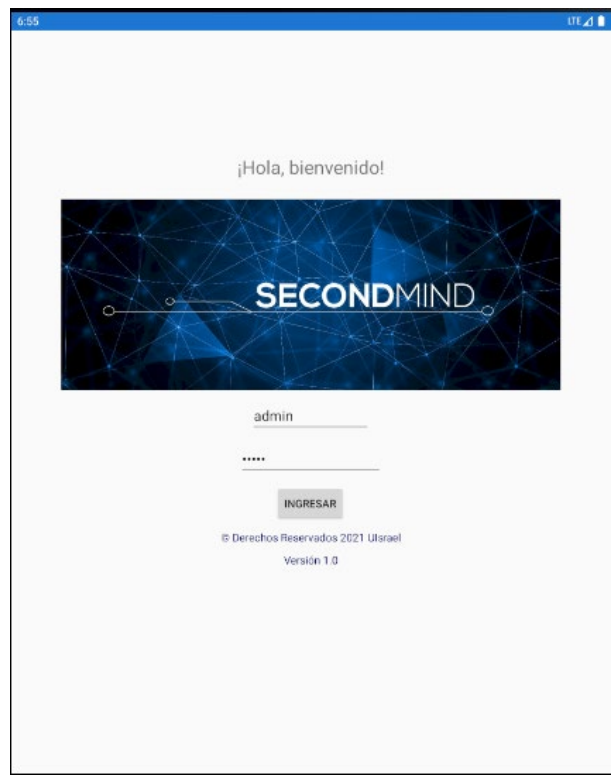

En la Figura 10 se muestra el menú con los dos botones (Registra/Recuerda) y en la Figura 11 se visualiza la pantalla para ingreso de información.

Figura 10.

Menú Principal

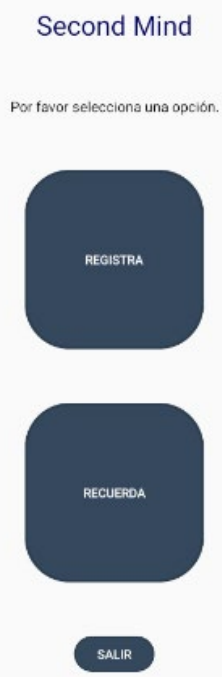

Figura 11.

Pantalla registro de memoria

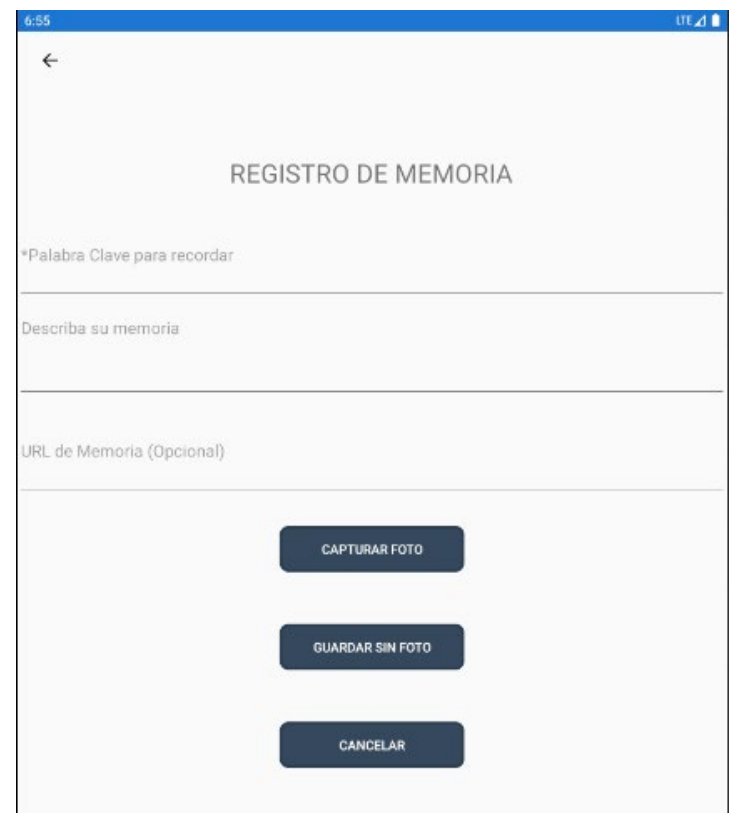


En la Figura 12 se ingresa los datos y en la Figura 13 se muestra la pantalla al seleccionar captura de imagen a ser registrada con los respectivos botones de control.

Figura 12.

Ingreso de información

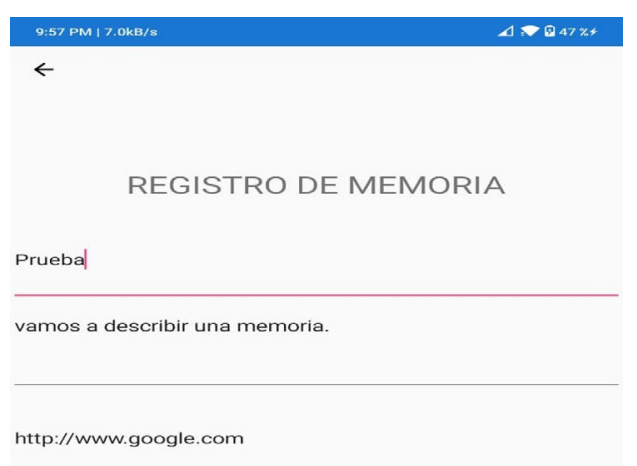

CAPTURAR FOTO

GUARDAR SIN FOTO

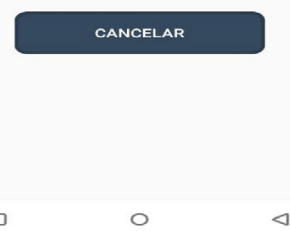

Figura 13.

Pantalla para capturar imagen

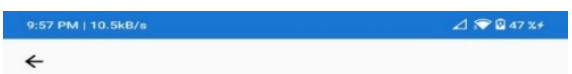

CAPTURAR UNA FOTO

SUBIR Y GUARDAR

VOLVER

En la Figura 14 se muestra en pantalla la imagen capturada con cámara de teléfono y en la Figura 15 se presenta el aviso para botón subir y guardar. 
Figura 14.

Pantalla de Imagen Capturada

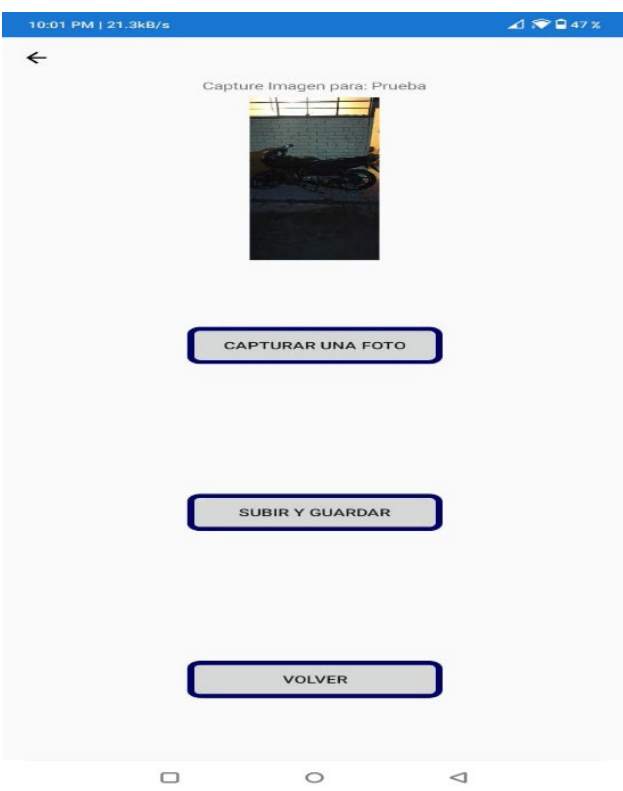

Figura 15.

Aviso por registro de imagen

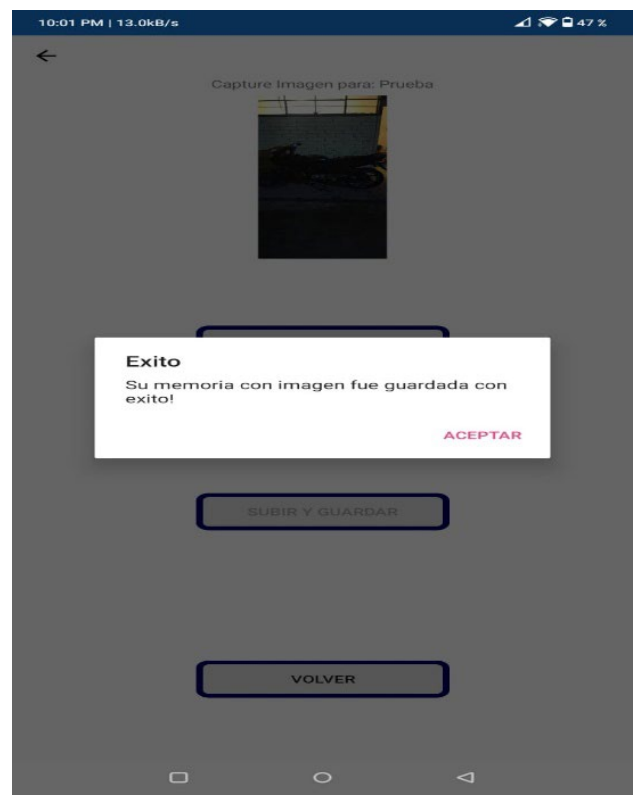

En la Figura 16 y Figura 17 se muestra la pantalla al seleccionar Recordar, donde se visualizan las memorias almacenadas y se consulta con la palabra clave.

Figura 16.

Acceso de botón recordar

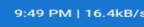

CONSULTA TU MEMORIA

Q Buscar

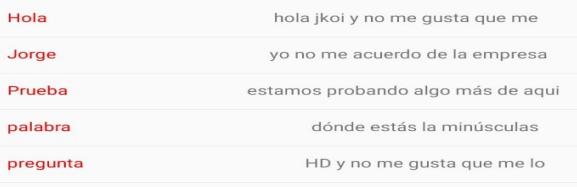

recuerdo
Figura 17.

Pantalla consulta con palabra clave
CONSULTA TU MEMORIA

a p

Prueba

palabra

pregunta

Prueba

Prueba estamos probando algo más de aqui

dónde estás la minúsculas
REGRESAR

口

o 
En la Figura 18 y Figura 19 se muestra la consulta hecha por el usuario, donde se visualiza la descripción, el texto y el enlace con el linkeo correspondiente.

Figura 18.

Resultado de consulta por descripción

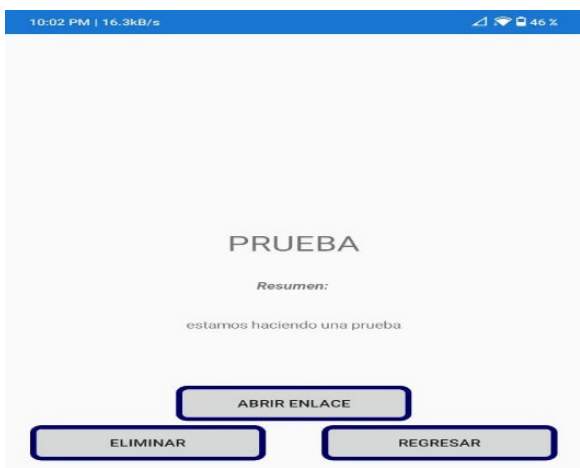

Figura 19.

Funcionalidad de acceso a url

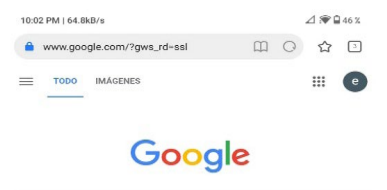

Google

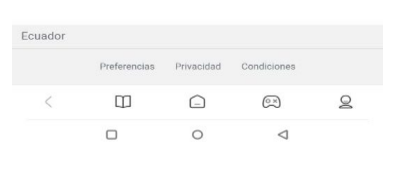

En la Figura 20 y Figura 21 se muestra la consulta hecha por el usuario, donde se visualiza la descripción, el texto y la imagen almacenada en repositorio, correspondiente a ese registro.

Figura 20.

Resultado de consulta por descripción

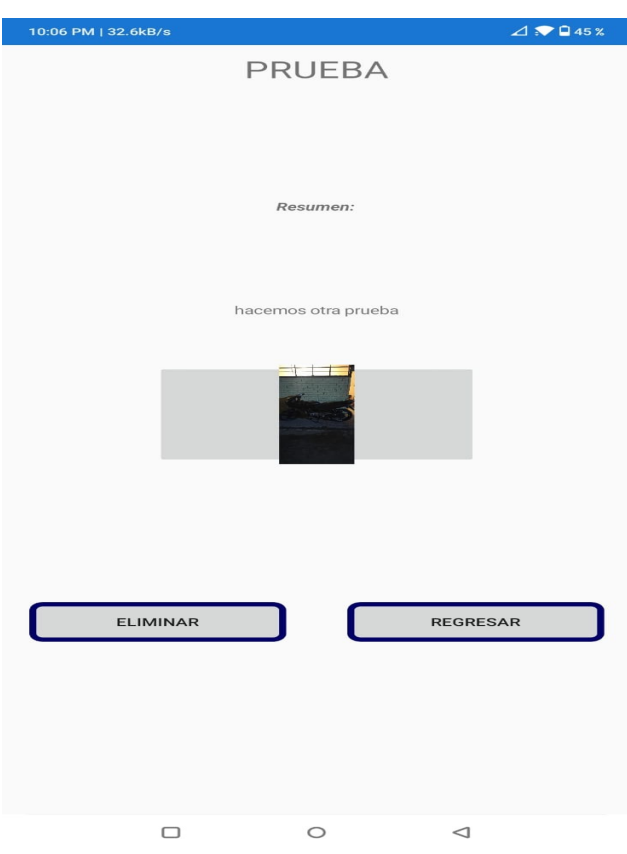

Figura 21.

Visualización de imagen en repositorio

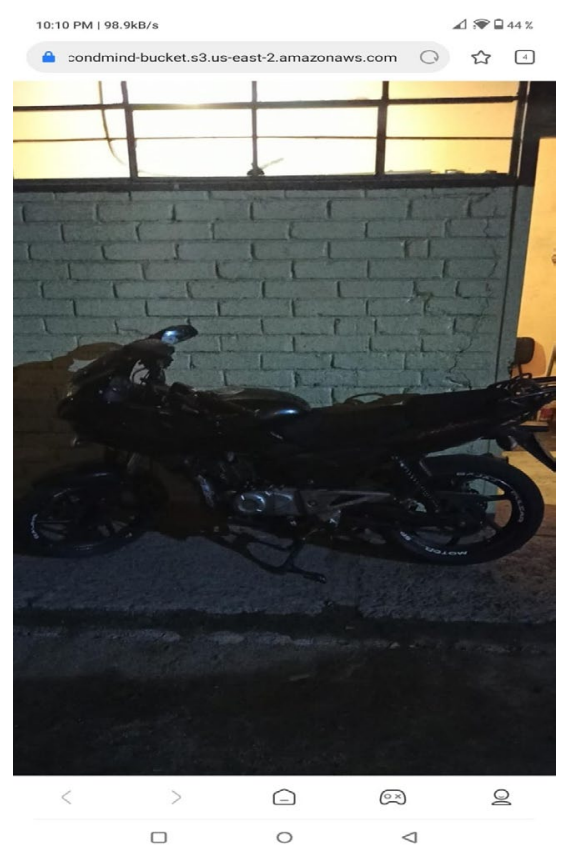


En la Figura 22 se puede evidenciar la funcionalidad de eliminación de un registro en aplicativo.

Figura 22.

Botón eliminar y mensaje de advertencia

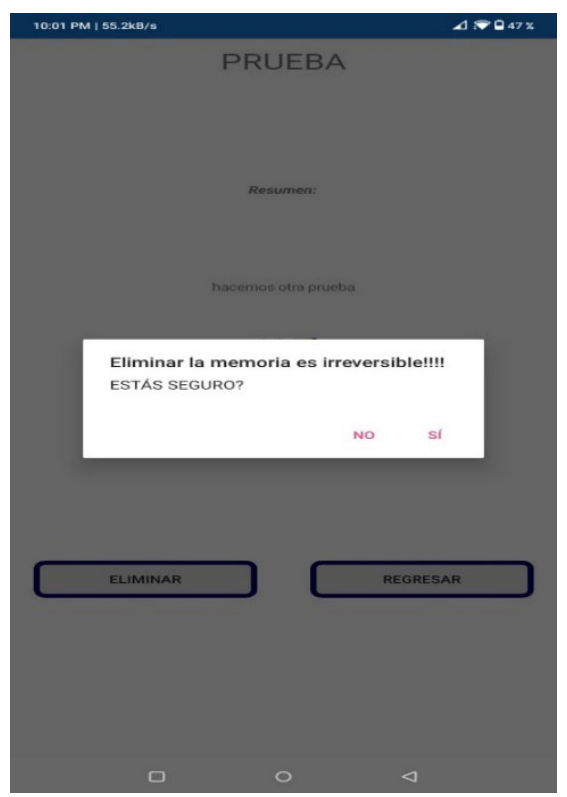

Finalmente, en las siguientes Figura 23, Figura 24 y Figura 25 se muestran las advertencias en caso de errores de ingreso, falta de información para registro y errores en ingreso de la URL.

Figura 23.

Error al ingresar

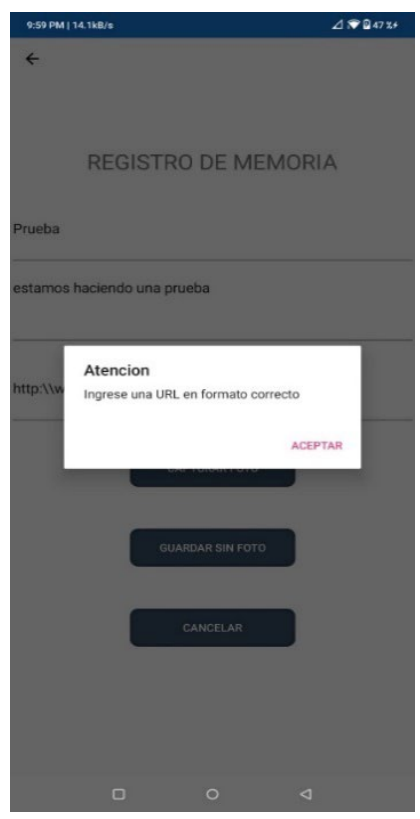

Figura 24.

Error al guardar sin información

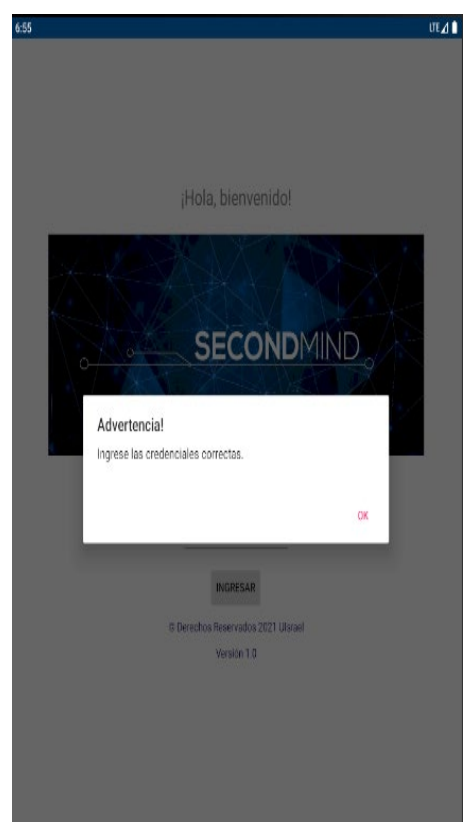

Figura 25.

Error de URL

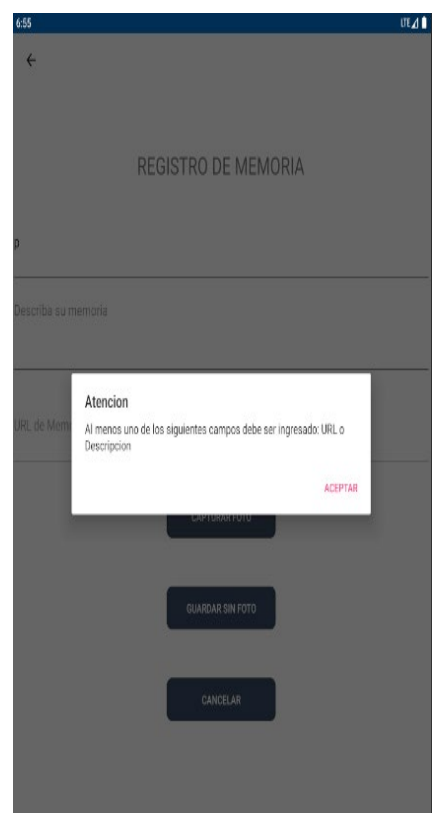




\section{Conclusiones}

Para el proyecto fue una parte primordial identificar la necesidad del usuario, así como también los requerimientos para que el prototipo sea efectivo, "Second Mind" es un aplicativo interactivo que permitirá registrar y almacenar información a corto o largo plazo, evitando así un almacenamiento caduco y tedioso, como es el uso innecesario de Google, o correos electrónicos para reenviarse y mantener la información, archivos en Pc que sean limitantes de acceder. Con tan solo el uso de esta app móvil, que tiene una interfaz amigable, se podrá disponer de textos, tutoriales y grabar incluso una URL o imágenes, usando su propia cámara del dispositivo, así traerá y recordará cualquier información que necesite de forma inmediata.

El almacenamiento de la información del prototipo se realizó de forma hibrida, la parte de texto en SQLite, por lo que la ubicación del archivo se especifica en la biblioteca y puede estar en su directorio de inicio en la carpeta del programa de invocación, y la imagen se almacena en el repositorio Amazon S3, por el tamaño que estas disponen, así el manejo de la persistencia de información fue exclusivo para el prototipo.

Para el desarrollo se utilizó Xamarin en Leguaje Visual Basic 2019, con una base SQLite y un repositorio Amazon S3 (proporciona almacenamiento de objetos a través de una interfaz de servicio web), como control de versiones el GitHub para agilizar la creación del prototipo y la metodología Kanban, para concluir dentro de los tiempos establecidos el desarrollo.

Finalmente, se realizaron las pruebas correspondientes, que permitieron verificar la funcionalidad del prototipo y que se cumplan los requerimientos funcionales y no funcionales, levantados al inicio del proyecto. 


\section{Referencias}

Amazon,W. (s.f). SDK de AWS para .NET. https://aws.amazon.com/es/sdk-for-net/

B, G. (08 de marzo de 2021). ¿Qué es GitHub y Cómo Usarlo? Hostinger Tutoriales. https://www.hostinger.es/ tutoriales/que-es-github

Develou. (s.f). Tutorial De Bases De Datos SQLite En Aplicaciones Android. https://www.develou.com/android-sqlite-bases-de-datos/

Dominguez, D, L. (26 de febrero del 2018). La revolución móvil (enfocada a las Apps). https://www.delaradominguez.es/apps-la-revolucion-movil/\#page-content

Gilibets, L. (11 de noviembre del 2020). Qué es la metodología Kanban y cómo utilizarla. IEBS. https://www. iebschool.com/blog/metodologia-kanban-agile-scrum/

Kanbanize. (s.f). Qué es Kanban: Definición, Características y Ventajas. https://kanbanize.com/es/recursos-de-kanban/primeros-pasos/que-es-kanban

Kawulich, B. (2005). Participant Observation as a Data Collection Method. Forum Qualitative Sozialforschung / Forum: Qualitative Social Research, 6(2). https://doi.org/10.17169/fqs-6.2.466

Madhuri, T., \& Sowjanya, P. (2016). Microsoft Azure v/s Amazon AWS cloud services: A comparative study. International Journal of Innovative Research in Science, Engineering and Technology, https://www.academia. edu/download/53932691/Microsoft Azure vs Amazon AWS Cloud.pdf

Madhuri, T., \& Sowjanya, P. (2016). Microsoft Azure v/s Amazon AWS cloud services: A comparative study. International Journal of Innovative Research in Science, Engineering and Technology, 5(3), 3904-3907. DOI:10.15680/IJIRSET.2016.0503098

Martín-Barbero, J. (2015). Comunicación masiva: discurso y poder (Vol. 1). Ediciones Ciespal.

Microsoft. (s.f ). Desarrollo de aplicaciones .NET. https://visualstudio.microsoft.com/es/vs/features/net-development/

Ruiz, J. (18 de diciembre de 2017). [Xamarin.Forms] Uso de SQLite, múltiples tablas, relaciones y operaciones en cascada. https://javiersuarezruiz.wordpress.com/2017/12/18/xamarin-forms-uso-de-sqlite-multiples-tablas-relaciones-y-operaciones-en-cascada/

SQLite, DB (09 de septiembre de 2018). SQLite Database search in Xamarin Forms https://stackoverflow.com/ questions/52241191/sqlite-database-search-in-xamarin-forms 
Copyright (c) 2022 Roberth Alexander Almache Vallejos, Jorge Ramiro Castro Chavez, Diego Alejandro González Calderon y Johanna Daniela Ruano Cardenas

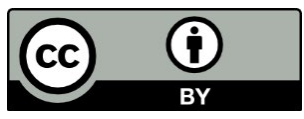

Este texto está protegido bajo una licencia internacional Creative Commons 4.0.

Usted es libre para Compartir-copiar y redistribuir el material en cualquier medio o formato - y Adaptar el documento - remezclar, transformar y crear a partir del material-para cualquier propósito, incluso para fines comerciales, siempre que cumpla las condiciones de Atribución. Usted debe dar crédito a la obra original de manera adecuada, proporcionar un enlace a la licencia, e indicar si se han realizado cambios. Puede hacerlo en cualquier forma razonable, pero no de forma tal que sugiera que tiene el apoyo del licenciante o lo recibe por el uso que hace de la obra.

Resumen de licencia - Texto completo de la licencia 